\title{
Highly sensitive carbon monoxide sensor for coal mine combustion monitoring
}

Zhaowei Wang, Yanfang Li, Tingting Zhang, Jie Hu, Yubin Wei, et al.

Zhaowei Wang, Yanfang Li, Tingting Zhang, Jie Hu, Yubin Wei, Tongyu Liu, "Highly sensitive carbon monoxide sensor for coal mine combustion monitoring," Proc. SPIE 11144, Photonics and Education in Measurement Science 2019, 1114417 (17 September 2019); doi: 10.1117/12.2530876

SPIE Event: Joint TC1 - TC2 International Symposium on Photonics and Education in Measurement Science 2019, 2019, Jena, Germany 


\title{
Highly sensitive carbon monoxide sensor for coal mine combustion monitoring
}

\author{
Zhaowei Wang*a , Yanfang $\mathrm{Li}^{\mathrm{a}}$, Tingting Zhang ${ }^{\mathrm{a}}$, Jie Hu${ }^{\mathrm{b}}$, Yubin $\mathrm{Wei}^{\mathrm{a}}$, Tongyu $\mathrm{Liu}^{\mathrm{a}}$ \\ ${ }^{a}$ Laser Institute, Qilu University of Technology (Shandong Academy of Sciences), Jinan, 250102,

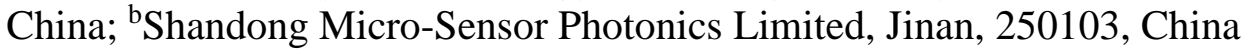

\begin{abstract}
As the most important characteristic gas, carbon monoxide $(\mathrm{CO})$ can be used for early detection of coal spontaneous combustion in mine goafs. Conventional gas analysis system for coal mine combustion monitoring is chromatographybased gas tubing bundles system, which suffers from long time delay. In this report, a sensitive and stable CO monitoring system was developed by using a distributed feedback (DFB) laser operating at $2.33 \mu \mathrm{m}$ and a Herriott-type multi-pass gas cell with a $20-\mathrm{m}$ optical length, taking advantage of the in-situ monitoring, excellent accuracy and simple structure available from direct absorption spectroscopy. The detection accuracy of system was about $\pm 0.2 \mathrm{ppm}$ when as low as 1 ppm CO gas was detected. And data monitored can be used to determine that the detection limit of system was about 0.2 ppm. Further, a long-term continuous monitoring evaluation has clearly demonstrated the long-term stability and reliability of the monitoring system. The results obtained have validated the potential use of such a $\mathrm{CO}$ monitoring system in a practical monitoring application, such as the coal spontaneous combustion monitoring.
\end{abstract}

Keywords: carbon monoxide, sensor, coal mine, combustion monitoring, TDLAS

\section{INTRODUCTION}

There are a great variety of health and safety hazards in the coal mines, such as water flooding, rock roof collapse, methane gas explosion, and coal mine combustion etc ${ }^{1}$. According to the published statistics, the coal mine combustion has been recognized as one of the major hazards in coal mine production, which can be classified as coal spontaneous combustion and electrical fault ${ }^{2}$. The coal spontaneous combustion is due to the slowly oxidized process of coal residue in the goaf. Because of the highly exothermic oxidized process, the great heat accumulated in turn accelerate the coal combustion and thus causes fire. The oxidization process is characterized by presence and increase of many kinds of gases $^{3}$, such as carbon monoxide $(\mathrm{CO})$ at initial phase of oxidization, ethene $\left(\mathrm{C}_{2} \mathrm{H}_{4}\right)$ when the oxidization gets severe, and ethyne $\left(\mathrm{C}_{2} \mathrm{H}_{2}\right)$ where its presence means fire is imminent.

So far, the fiber optic Raman scatter-based distributed temperature sensor (DTS) is an effective approach for coal spontaneous combustion monitoring, which provides a convenient means to monitor the temperature distribution of coal area over time ${ }^{4}$. However, the DTS fiber cable cannot be directly mounted in coal oxidization zone. Hence, the monitored temperature by the DTS technology is not an actual temperature of coal oxidization zone. More recently, multi-gas monitoring technology has attracted more and more attention to forecasting coal spontaneous combustion ${ }^{5}$. By monitoring the concentrations of each gas within the mixed gases, the early warning signal can be issued before coal spontaneous combustion. Conventional gas analysis system for coal mine combustion monitoring is chromatographybased gas tubing bundles system ${ }^{6}$, which suffers from long time delay due to the longer distance (10 km or longer) between goaf and the underground gas analysis system. As a cutting-edge technology for gas detection, tunable diode laser absorption spectroscopy (TDLAS) shows the advantages and potential for real time on-line monitoring, offering high selectivity and sensitivity, long-term stability and excellent reliability ${ }^{7}$. As is known, TDLAS technique can be classified into two major method: conventional direct absorption spectroscopy and wavelength modulation spectroscopy. The former technique allows a more reliable sensor with the simple and compact structure to be developed, and this approach was employed in this paper.

*zhaowei.wang@sdlaser.cn; www.sdlaser.cn

Photonics and Education in Measurement Science 2019, edited by Maik Rosenberger,

Paul-Gerald Dittrich, Bernhard Zagar, Proc. of SPIE Vol. 11144, 1114417

(C) 2019 SPIE $\cdot$ CCC code: 0277-786X/19/\$21 · doi: 10.1117/12.2530876 
Among these characteristic gases, $\mathrm{CO}$ is the most important gas for early detection of coal spontaneous combustion ${ }^{8}$. Typical detection sensitivity of CO present in goaf area is 1 part per million (ppm) by volume. Because of the stronger absorption of the $\mathrm{CO}$ first overtone band, a $\mathrm{CO}$ sensor using a laser diode operating around $2.3 \mu \mathrm{m}$ can provide sensitive detection. Therefore, in this paper, a sensitive (sub-ppm level) and reliable CO monitor has been demonstrated by using a $2.33 \mu \mathrm{m}$ laser diode, taking advantage of real time on-line monitoring, excellent accuracy, simple structure and lower cross interference available from direct absorption spectroscopy.

\section{SYSTEM STRUCTURE AND PRINCIPLE}

A schematic of the CO gas monitoring system is shown in Figure 1. A distributed feedback (DFB) laser operating at a specific wavelength of $2330.18 \mathrm{~nm}$ was employed to target the strong absorption line of CO first overtone band, a wavelength where the corresponding absorption intensity is $3.39 \times 10^{-21} \mathrm{~cm}^{-1} /\left(\mathrm{mol} \times \mathrm{cm}^{-2}\right)$. The Herriott-type multi-pass gas cell (MGC) has an effective optical length of $20 \mathrm{~m}$, with a base volume of $0.57 \mathrm{~L}$. Both sides of the inside of the gas cell were dielectric coated, providing a broadband transmission over the wavelength range from $2290 \mathrm{~nm}$ to $2370 \mathrm{~nm}$. The transmitted laser beams were detected by using the RT extended-InGaAs photo-detector installed within the system. Using a current driver and temperature controller, the emitting laser wavelength can be tuned with a sawtooth wave. The intensity of the laser decreases when the laser light is passed through CO gas-filled MGC and as a result, the measured small dip in the background line creates the gas absorption signal. According to the Lambert-Beer law ${ }^{9}$, the CO gas concentration can be obtained using following formula:

$$
\begin{gathered}
C=\frac{A}{P S(T) L} \\
A=\int_{-\infty}^{\infty}-\ln \left(\frac{I}{I_{0}}\right) d \lambda
\end{gathered}
$$

where $\mathrm{C}$ is the volume concentration of the measured $\mathrm{CO}$ gas sample, $I_{0}$ is the initial light intensity, $I$ is the light intensity due to the absorption, $\lambda$ is the laser wavelength, $L$ is the length of the optical path, $P$ is the total pressure of the gas medium and $S(T)$ is the intensity of the characteristic spectral lines (which show a temperature dependence).

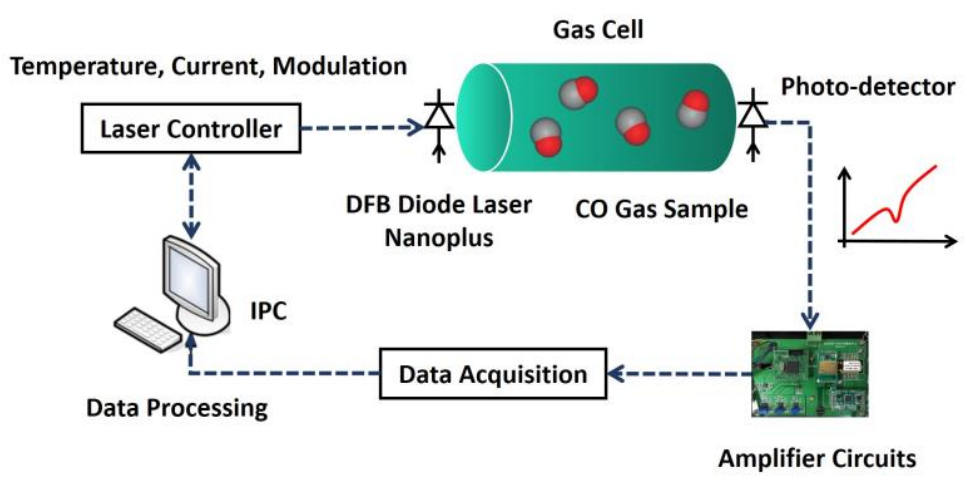

Figure 1. Schematic of the CO gas monitoring system developed.

\section{USER INTERFACE AND PROTOTYPE}

As shown in Figure 2, the user interface for the CO monitoring system was developed by using LabVIEW software and comprises three key parts: real-time data for the $\mathrm{CO}$ concentration, the operating parameters of the system and the 
detected signals represented graphically. The system was designed to display to the user the key control parameters and the 'run data' from the $\mathrm{CO}$ detection, together with displaying the graphs showing the changes in the detected signals, the normalized signals, the nonlinear least-squares fitted curve and thus a real-time measured value of the detected $\mathrm{CO}$ concentration. The engineering prototype of $\mathrm{CO}$ monitoring system was successfully developed for the further testing, as depicted in Figure 3.

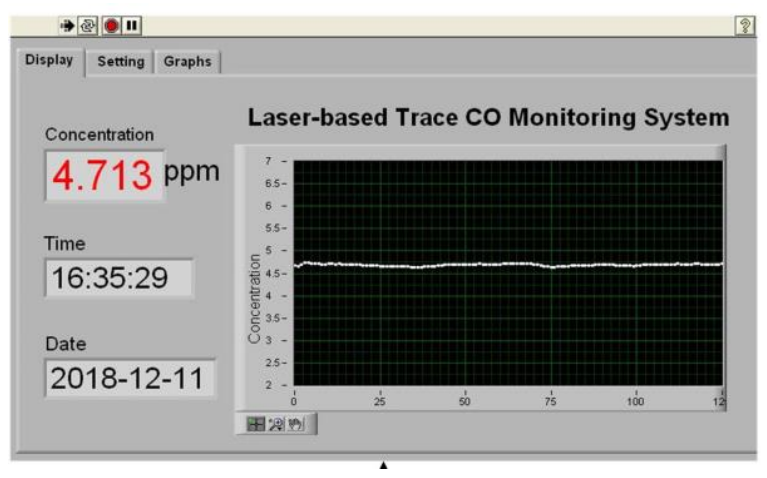

Figure 2. User interface developed using LabVIEW software.

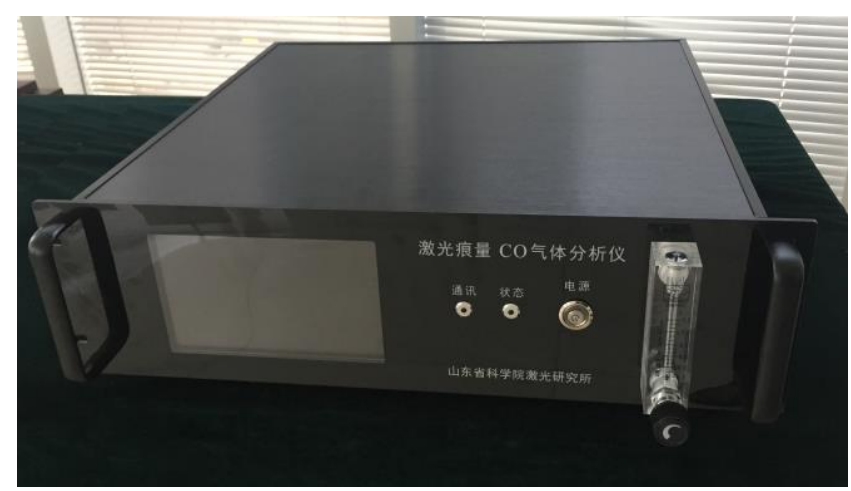

Figure 3. The engineering prototype of $\mathrm{CO}$ monitoring system.

\section{RESULTS AND DISCUSSION}

\subsection{Performance of system}

In the experiment carried out, the $\mathrm{CO}$ gas, at a concentration of $30 \mathrm{ppm}$, was mixed with pure nitrogen $\left(99.999 \% \mathrm{~N}_{2}\right)$ to create various $\mathrm{CO}$ gas samples at different nominal concentrations: $3 \mathrm{ppm}, 1 \mathrm{ppm}, 0.5 \mathrm{ppm}, 0.3 \mathrm{ppm}$, and $0.1 \mathrm{ppm}$. These gases were successively filled into the gas cell with a constant flow rate of $1000 \mathrm{~mL} / \mathrm{min}$. The monitored raw data were recorded during the whole process and these are illustrated in Figure 4. At the same time, the measured raw data were smoothed using a 10-points moving average method, in order to show the trends more clearly. As depicted, the results show that the CO monitoring system could detect CO gas samples with excellent precision and stability. And it can readily sense the presence of $\mathrm{CO}$ gas, at a concentration as low as $0.1 \mathrm{ppm}$. As illustrated in Figure 4, data monitored can be used to determine that the detection accuracy of system was about $\pm 0.2 \mathrm{ppm}$ when as low as $1 \mathrm{ppm} \mathrm{CO}$ gas was detected and the detection limit of system could be estimated to be $0.1 \mathrm{ppm}$ in this work. Due to extremely small amount of impurity of pure $\mathrm{N}_{2}$, the measurement accuracy of $\mathrm{CO}$ monitoring system decreased when $0.2 \mathrm{ppm} \mathrm{CO}$ gas was detected. 


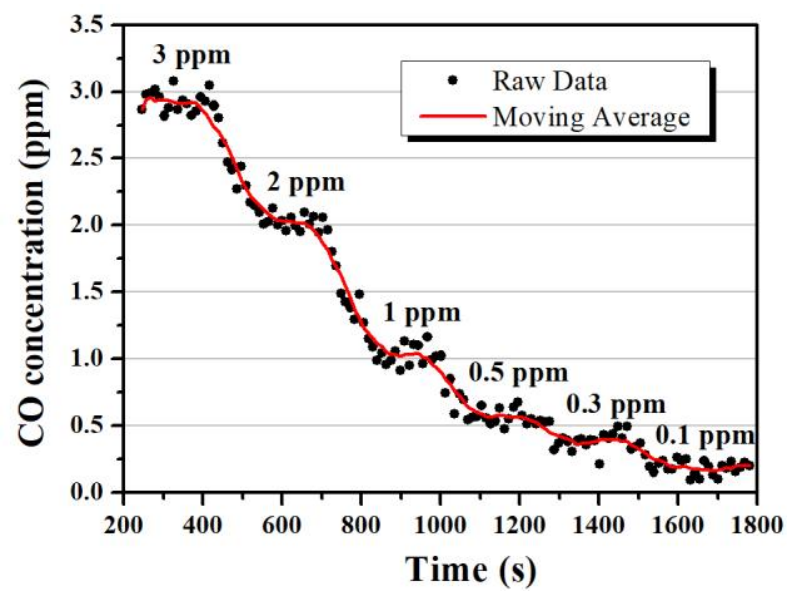

Figure 4. Measured CO concentrations determined from the monitoring system developed.

\subsection{Field test}

To evaluate the stability and reliability of CO monitor developed, the system operated continuously for over 600,000 second in an ambient atmosphere, as shown in Figure 5. In order to enhance the stability of system, the measured raw data were smoothed using a 30-points moving average method. As can be seen from the Figure 5, the actual fluctuations of the $\mathrm{CO}$ gas concentration in the ambient air could be measured by using the $\mathrm{CO}$ monitoring system developed. Looking at these closely, the first 'rogue peak' resulted from the leaking $\mathrm{CO}$ gas from another experiment being carried out in the same place. Figure 5 has clearly demonstrated the long-term stability and reliability of monitoring system. More importantly, there is no cross interference seen in the monitoring of ambient air. The results obtained have validated the potential use of such a $\mathrm{CO}$ monitoring system in a practical monitoring application, such as the coal spontaneous combustion monitoring.

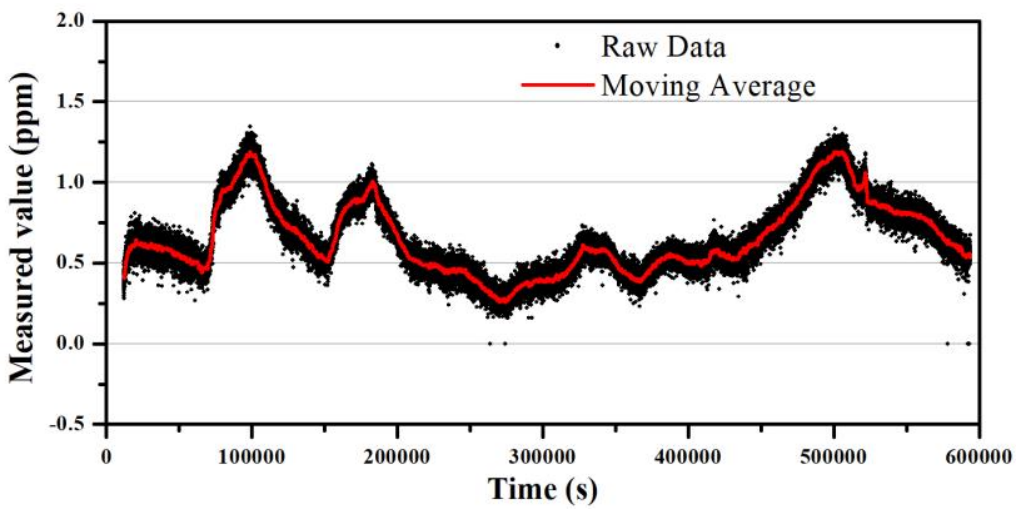

Figure 5. The measured $\mathrm{CO}$ concentration using the monitoring system developed.

\section{CONCLUSION}

In summary, multi-gas monitoring technique is an effective approach for coal spontaneous combustion monitoring, in addition to fiber optic Raman scatter-based DTS. Conventional gas analysis system for coal mine combustion monitoring is chromatography-based gas tubing bundles system, which suffers from long time delay. As a cutting-edge technology for gas detection, tunable diode laser absorption spectroscopy (TDLAS) shows the advantages and potential for real time on-line monitoring, offering high selectivity and sensitivity, long-term stability and excellent reliability. 
As the most important characteristic gas, $\mathrm{CO}$ gas can be used for early detection of coal spontaneous combustion in mine goafs. In this report, a sensitive and stable CO monitoring system was demonstrated by using a $2.33 \mu \mathrm{m}$ distributed feedback (DFB) laser and a 20-m Herriott-type multi-pass gas cell, taking advantage of in-situ monitoring, excellent accuracy, simple structure and lower cross interference available from direct absorption spectroscopy. The detection accuracy of system was about $\pm 0.2 \mathrm{ppm}$ when as low as $1 \mathrm{ppm}$ CO gas was detected. Data monitored can be used to determine that the detection limit of system was about $0.2 \mathrm{ppm}$. Moreover, a long-term continuous monitoring evaluation has clearly demonstrated the excellent stability and reliability of monitoring system developed. The results obtained have validated the potential use of such a $\mathrm{CO}$ monitoring system in a practical monitoring application, such as forecasting coal spontaneous combustion.

\section{ACKNOWLEDGEMENT}

The authors gratefully acknowledge the support by the National Key Research and Development Program of China (2017YFC0804403), Natural Science Foundation of Shandong Province (ZR2016QZ006), Young Fund Project of Shandong Academy of Sciences (2018QN0011), and Young Scientist Partnership Project between Chinese Academy of Sciences at Shenyang and Shandong Academy of Sciences.

\section{REFERENCES}

[1] Y. Liang, X. Hou, H. Luo, F. Tian, G. Yu, "Development countermeasures and current situation of coal mine fire prevention \& extinguishing in China," Coal Science \& Technology 44(6), 1-6 (2016)

[2] Lieberman H, Hewitt C., "Innovative Techniques for Detection and Control of Underground Spontaneous Combustion of Coal," Communications of the Acm. 26(6), 419-429 (2004)

[3] Liang Y. T., Luo H. Z., "Current situation and development trend for coal mine fire prevention \& extinguishing techniques in China" Journal of China Coal Society 33(2), 126-130 (2008).

[4] Liu Y., Tao L., Wei Y., et al. "Application of distributed optical fiber temperature sensing system based on Raman scattering in coal mine safety monitoring," Symposium on Photonics and Optoelectronics. IEEE, 1-4 (2012).

[5] X. H. Zhang, H. Wen, J. Deng, X. C. Zhang, J. C. Tien, "Forecast of coal spontaneous combustion with artificial neural network model based on testing and monitoring gas indices," Journal of Coal Science \& Engineering 17(3), 336-339 (2011).

[6] Z. R. Jr, W. Marchewka, K. Mohamed, J. Addis, F. Karnack, "Tube bundle system for monitoring of coal mine atmosphere," Min. Eng. 65(5), 57-63 (2013).

[7] Hodgkinson J, Tatam R. P., "Optical gas sensing: a review," Measurement Science \& Technology 24(1), 012004 (2013).

[8] Qi-Ling He, Guang-Long Dai, De-Ming Wang, "Influence of Moisture on Absorbed Oxygen and Released Heat of Coal," Journal of China University of Mining \& Technology 34(3), 358-362, (2005).

[9] Paynter R. W., "Modification of the Beer-Lambert equation for application to concentration gradients," Surface \& Interface Analysis 3(4), 186-187 (2010). 